\title{
Cardiogenic shock following hydrogen sulfide poisoning
}

\author{
lianlian Jiang ${ }^{1}$, Jie Jiang ${ }^{1}$, and Xi Chen ${ }^{1}$ \\ ${ }^{1}$ Southeast University Zhongda Hospital
}

November 11, 2020

\begin{abstract}
Hydrogen sulfide (H2S) is an asphyxiating industrial poison, which is likely to cause serious damage to myocardial function and even cause cardiogenic shock after poisoning. The injury is insidious and tardive but the consequences are serious, so it needs to be prevented and treated in time. We herein present a case of an otherwise healthy 51-year-old man who developed into cardiogenic shock dynamically after poisoning and treated successfully with IABP and VA-ECMO.
\end{abstract}

\section{Cardiogenic shock following hydrogen sulfide poisoning}

ORCID

Lian-Lian Jiang https://orcid.org/0000-0002-0908-3864

Lian-Lian Jiang $\mathrm{MB}^{1} \mid$ Jie Jiang $\mathrm{MM}^{2} \mid \mathrm{Xi}$ Chen $\mathrm{MM}^{3}$

${ }^{123}$ Jiangsu Provincial Key Laboratory of Critical Care Medicine, Department of Critical Care Medicine, Zhongda Hospital, School of Medicine, Southeast University, Nanjing,210009, China

Correspondence

Lian-Lian Jiang, Department of Critical Care Medicine, Zhongda Hospital, School of Medicine, Southeast University, Nanjing, 210009,China.

Email:lianlianjiang2020@163.com

CONFLIC T OF INTEREST

The authors report no conflicts of interest related to the work described in this manuscript.

\section{Abstract}

Hydrogen sulfide $\left(\mathrm{H}_{2} \mathrm{~S}\right)$ is an asphyxiating industrial poison, which is likely to cause serious damage to myocardial function and even cause cardiogenic shock after poisoning. The injury is insidious and tardive but the consequences are serious, so it needs to be prevented and treated in time. We herein present a case of an otherwise healthy 51-year-old man who developed into cardiogenic shock dynamically after poisoning and treated successfully with IABP and VA-ECMO.

\section{Keywords}

cardiac toxicity, cardiogenic shock, intra-aortic ballon pump(IABP), veno-arterial extracorporeal membrane oxygenation(VA-ECMO), ejection fraction

\section{1 | Introduction}


Hydrogen sulfide $\left(\mathrm{H}_{2} \mathrm{~S}\right)$ remains a chemical hazard in natural gas and agriculture, which is prone to pose a potential threat to the civilian population. The damage of respiratory and brain caused by hydrogen sulfide never surprises us any more ${ }^{1}$, but cases related to myocardial injury have rarely been reported so far. Especially, existing articles are in lack of pathological judgment of myocardium.

\section{2| Case report}

A 51-year-old man was trapped in a closed sewage tank for about 20 minutes during his project on August 6. He was in coma when pulled out without electrocardiographic abnormality(Figure 1A). Endotracheal intubation, and pure oxygen inhalation were arranged immediately. The patient has no significant medical background or family history. After treatment, His consciousness became clear gradually. However, electrocardiogram showed extensive elevation of ST segment (Figure 1B)and myocardial enzyme index were increased dramatically on August ${ }^{\text {th }}$. What's more, ventricular fibrillation occurred on August $9^{\text {th }}$. Luckily, sinus rhythm and consciousness was restored about one minute after cardiopulmonary resuscitation. No obvious abnormality was found in coronary computed tomographic angiography (CTA), but pluse indicator continuous cardiac output (PICCO) suggested low cardiac output with $1.9 \mathrm{~L} / \mathrm{min} / \mathrm{m}_{2}$ of the cardiac index (CI).

Cardiotonic drugs like levosimendan were not effective, so intra-aortic ballon pump (IABP) was implemented then. The ST segment decreased to normal with left ventricular ejection fraction(EF) increased to $46 \%$ on August $13^{\text {th }}$ and then the IABP was removed. However, he slipped back into a coma with ST segment elevated and EF decreased to $31 \%$ again afterwards. So veno-arterial extracorporeal membrane oxygenation (VAECMO) was implemented. Two days later, his ST segment elevated again (Figure 1C) with extremely bad heart function (Movie S1). Coronary angiography(CAG) found no stenosis but cardiomyocyte hypertrophy was showed in the pathology of myocardial biopsy(Figure 2A) and mitochondrial swelling, dissolution as well as vacuolization were observed under electron microscopy(Figure 2B). In order to support heart function powerfully, we put IABP to cooperate with VA-ECMO.

After about 2 weeks' support of V-A ECMO and 3 of IABP, his heart function gradually returned to normal without any support(Figure 1D,Figure 3, Figure 4).

\section{3 | Discussion}

$\mathrm{H}_{2} \mathrm{~S}$ is considered to be a mitochondrial poison. It inhibits cytochrome $\mathrm{C}$ oxidase, blocks the activity of the mitochondrial electron transport chain, disrupts the aerobic metabolism of cells, hinders the production of ATP, and ultimately leads to reduced oxygen use of cardiomyocytes and myocardial damage. ${ }^{2}$ Besides, $\mathrm{H}_{2} \mathrm{~S}$ poisoning can also rapidly reduce cardiac contractility and cardiac output, leading to cardiogenic shock even cardiac arrest within a few minutes, possibly due to the blocking of the L-type calcium channel of myocardial cells. ${ }^{3}$

Several autopsies showed that the skin and internal organs of these patients had green discoloration with pulmonary edema. ${ }^{4}$ Our report supplements that their myocardial tissue experienced sarcolysis and hypertrophy with increased intracellular vacuoles, irregular nuclei and swelling mitochondrial.

Delayed myocardial damage can occur in a few days after exposure, and clinical manifestations include STsegment elevation, increased myocardial markers, decreased ventricular wall motion, myocardial infarction , arrhythmia, etc. ${ }^{5}$ It can develop within a few weeks and most of them died of circulatory failure caused by acute inhibition of myocardial contractility. We successfully helped the patient go through this acute stage with the support by combination of IABP and VA-ECMO. As we know, this case is the first report of using IABP and VA-ECMO in cardiogenic shock caused by $\mathrm{H}_{2} \mathrm{~S}$ successfully.

\section{4 | conclusion}

Cardiac changes in patients with hydrogen sulfide poisoning are delayed and abrupt, which is difficult to maintain by conservative treatments. However, returning to normal cardiac function is still possible when providing strong cardiopulmonary support like IABP and VA-ECMO during the transition. 


\section{REFERENCES}

1. Haouzia P, Sonobea T, Judenherc-Haouzib A. Hydrogen sulfide intoxication induced brain injury and methylene blue. Neurobiology of Disease. 2020;133:104474.

2. Maebashi K, Iwadate K, Sakai K , et al. Forensic Science International. 2011;207: 91-95.

3. Sonobe T, Haouzi P. Sulfide Intoxication-Induced Circulatory Failure is Mediated by a Depression in Cardiac Contractility. Cardiovasc Toxicol. 2016;16:67-78.

4. Ago M, Ago K, Ogata M. Two fatalities by hydrogen sulfide poisoning: Variation of pathological and toxicological findings. Legal Medicine. 2008;10:148-152.

5. Mo WC, Shen J, Huang XC, et al. Acute myocardial injury following hydrogen sulfide poisoning. Toxicology and Industrial Health. 2020.

\section{FIGURE LEGEND}

FIGURE $1 \mathrm{~A}$ and D, The electrocardiogram of them were both normal; B showed extensive elevation of ST-T in all the leads and ST-T of C was seen to be elevated in lead I, II, III, aVR, aVF and V3, V4, V5, and $\mathrm{V} 6$.

FIGURE 2 A, Myocardial biopsy showed cardiomyocyte hypertrophy; B, Disorders of myocardial fibers, tissue sarcolysis and hypertrophy, irregular nuclei as well as swelling and dissolved mitochondria (arrows) were observed under electron microscope.

FIGURE 3 EF showed in the echocardiography returned to normal. LV, left ventricle; LA, left atrium; RA, right atrium.

FIGURE4 Cardiac contrast-enhanced magnetic resonance images showed nothing wrong with its perfusion and signal.

Movie S1. Reduced diffuse cardiac activity could be seen in the echocardiography. Its video coder unit was MPEG-4.

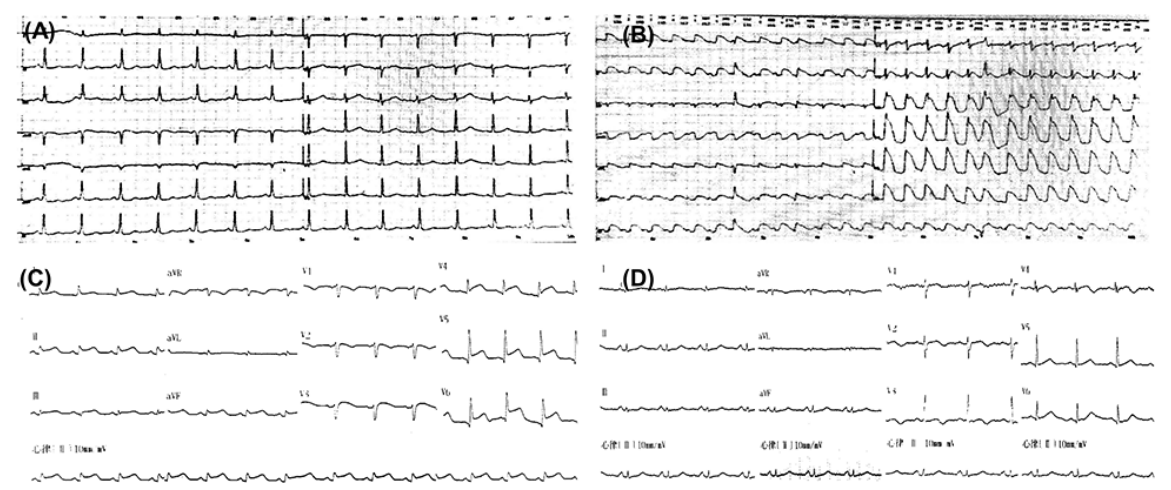



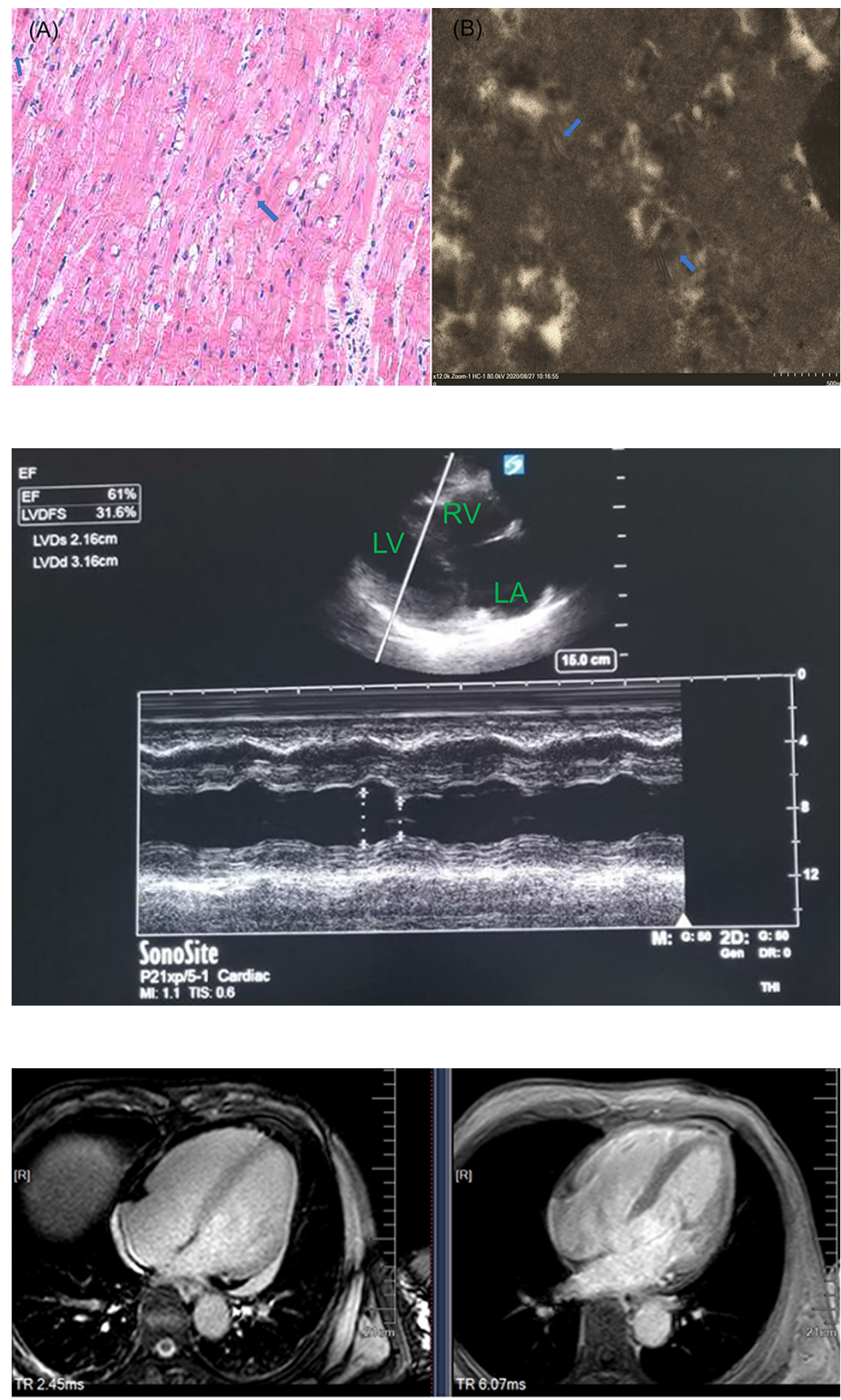